\title{
Variability of gastrointestinal transit in healthy women and men
}

\author{
L P Degen, S F Phillips
}

\begin{abstract}
Background and Aims-Measurements of gastrointestinal transit are made in clinical and research gastroenterology, yet their intrinsic variability is not well characterised. In particular, an influence of hormones on transit has been proposed as the basis for gastrointestinal symptoms that vary with the menstrual cycle. Our aims were to quantify individual differences in transit during the menstrual cycle in healthy women and to compare these with the intrinsic variability in healthy men.

Methods-On two occasions, whole gut transit was asssessed scintigraphically and colonic transit quantified by radioopaque markers. Thirty two healthy volunteers (12 women, 20 men) were studied, women during the follicular and luteal phases, men twice within a similar four week period. Diets and exercise were standardised prior to and during both studies.
\end{abstract}

Results-Colonic transit was significantly faster in men, and postlag gastric emptying was also more rapid; other indices of regional transit were not different between the sexes. Total colonic transit time was equally well reflected by the scintigraphic and radio-opaque marker methods. Important intraindividual differences were noted in both sexes. The variances in our samples predicted an $80 \%$ chance of detecting (with $95 \%$ confidence) a mean effect of menstrual hormones on transit that was in the same range as the intrinsic variation in men.

Conclusions-Colonic transit was faster in men than in women. Although group means in the two studies were almost identical, single assessments of transit in subjects sometimes exhibited considerable variability, implying broad biological variations. Given this intrinsic variability, the influence of menstrual hormones on gastrointestinal transit must be small and of doubtful clinical significance.

(Gut 1996; 39: 299-305)

Keywords: transit, reproducibility, sex differences.

Rates at which food and digestive products traverse the gastrointestinal tract reflect the integrated activity of intestinal smooth muscle, are thought to be relevant to certain symptoms, ${ }^{1-3}$ and have been quantified by several methodologies. ${ }^{1-7}$ Scintigraphic assessments of gastric emptying are perhaps best established, and they are now standard in many nuclear medicine facilities; reports of their reproducibility have been published. ${ }^{8-10}$ Scintigraphy has now been applied also to small bowel and colonic transit ${ }^{235}$ and the techniques have been simplified enough to make clinical tests for whole gut transit feasible. ${ }^{11-13}$ However, the day to day reproducibility of small bowel and colonic transit have not been reported, even though these indices are required for adequate interpretation of clinical and experimental studies. Moreover, bowel habits may vary daily, especially in patients with functional gastrointestinal disorders. It is important, therefore, that the normal biological variability of gastrointestinal transit be documented.

Despite longstanding interest in their influence on bowel habits, the effects of the menstrual hormones on gut function remain quite uncertain. The influences of the sex steroids on smooth muscle function and motility in animals is well substantiated, ${ }^{14-18}$ but comparable effects have never been well substantiated in humans. Thus, the results of earlier studies are in conflict; some propose a significant influence, ${ }^{19-24}$ or no effect, ${ }^{25-30}$ of the menstrual cycle on gastrointestinal transit. On the other hand, gastrointestinal symptoms often attributed to disorders of motility, have been reported to vary with the menstrual cycle. ${ }^{31-33}$

Our non-invasive scintigraphic method 235 11-13 provides a simple, comprehensive, and noninvasive tool by which transit of solid material through the entire, unprepared gastrointestinal tract can be measured. Our aim, therefore, was to quantify the differences in gastrointestinal transit in healthy women during the luteal and follicular phases of one menstrual cycle and to compare intraindividual differences in gastrointestinal transit between women and men.

\section{Methods}

EXPERIMENTAL SUBJECTS

Twelve healthy women and 20 healthy men, aged between 19 and 45 years, were recruited by public advertisement. None complained of gastrointestinal symptoms, or had a history of gastrointestinal disease or surgery other than appendicectomy or herniorrhaphy. Functional bowel symptoms were specifically excluded by evaluating the Manning criteria. ${ }^{34}$ Volunteers with acute symptoms or current use of drugs known to change gastrointestinal motility, and women taking birth control pills or using an intrauterine device within the last six months, were excluded. Persons with a body mass index over $30 \mathrm{~kg} / \mathrm{m}^{2}$ were also excluded 
because transit may be altered in clinically obese patients. ${ }^{35-37}$ In addition women must have had a regular menstrual cycle for the last six months, the length needed to be $28 \pm 4$ days and there had to be no major symptoms during the menstrual cycle. Excluded also were lactating women and those with a pregnancy within the last year.

With standardised questions, the smoking habits as well as the consumption of alcohol and coffee were assessed. After discussion of the procedure in detail, written consent was obtained for a protocol approved previously by the Institutional Review Board and the Radiation Control Committee of Mayo Clinic. All female volunteers had to have had a negative plasma $\beta$ human chorionic gonadotrophin pregnancy test no longer than 48 hours before each scintigraphic study.

\section{EXPERIMENTAL PROTOCOL}

Three days prior to and during the entire study, volunteers ingested a weight maintaining diet, based on the Harris-Benedict equation, ${ }^{38}$ with adjustment for daily physical activity. Volunteers were asked to avoid unusually intensive physical activity. Meals were provided by the Mayo General Clinical Research Center and their composition was normalised to $53 \%$ carbohydrate, $17 \%$ protein, and $30 \%$ fat. Fibre intake was standardised to 15 gram per day, consisting of $60 \%$ (9 gram) water insoluble and 40\% (6 gram) water soluble fibre. ${ }^{39}$

On each of days 2-4, volunteers ingested at 9 am a capsule containing 24 radioopaque markers (SITZMARKSÔ, Lafayette Pharmacal, 4200 South Hulen Street, Fort Worth, TX 76109). At 9 am on day four, an abdominal $x$ ray was obtained for assessment of the distribution of radio-opaque markers. ${ }^{7}$ On day four at 7 am, after fasting since midnight, the scintigraphic transit study begun and it finished 48 hours later.

At the beginning of each study period, all participants reported their physical activity within the last week, corresponding to the Harvard Alumni Activity Survey questionnaire ${ }^{40}$ and completed the self report inventory SCL-90- $\mathrm{R}^{41}$ to reflect their pattern of psychological symptoms within the past week. Immediately before the transit study, a venous blood sample was draw in women, to measure the concentrations of progesterone and oestradiol.

Women had two studies based on the menstrual cycle. Day 1 of the cycle was defined as the first day of menstrual flow. One transit study was performed on day 7-10 (=follicular phase) and one on day 21-24 (=luteal phase). At random, seven women had the first study during the follicular and five during the luteal phase. Men had the study repeated at equivalent times; an initial assessment was followed by the second within 14-17 days.

\section{Study procedure}

Gastric, small bowel, and colonic transit was measured by the non-invasive scintigraphic method developed in our laboratory. $23511-13$ Briefly, polystyrene Amberlite 120-IR-Plus resin pellets (average diameter $1 \mathrm{~mm}$; range $0.5-1.8 \mathrm{~mm}$ ) were labelled in an acid medium with $100 \mu \mathrm{Ci}$ of ${ }^{111} \mathrm{In} \mathrm{Cl}_{3} .{ }^{42}$ The efficiency of the labelling was $>98 \%$, as judged by thin layer chromatography. A capsule filled with approximately $0.5 \mathrm{~g}$ pellets and coated with one layer of methacrylate was given to fasting volunteers. As expected, the capsule dissolved in the ileocaecal region and thereafter marked ileocaecal transfer and colonic transit of contents.

External radioactive markers were placed over both anterior superior iliac spines to estimate the location of the capsule. As soon as the radiolabelled capsule passed into the small bowel, a breakfast was ingested within five minutes. It consisted of two scrambled eggs, one slice of whole wheat bread, and skimmed milk (35\% protein, $52 \%$ carbohydrate, $13 \%$ fat, $219 \mathrm{kcal})$. The scrambled eggs were mixed and cooked with $1 \mathrm{mCi}$ of ${ }^{99 \mathrm{~m}} \mathrm{Tc}$ labelled Amberlite 410 resin pellets (average diameter $1 \mathrm{~mm}$ ) to a firm consistency to provide a solid medium. These pellets had been labelled with ${ }^{99} \mathrm{Tc}$ sodium pertechnetate in a neutral medium. ${ }^{42}$

Four hours after breakfast, a standardised non-radiolabelled lunch (chicken, potato, butter, tapioca pudding, and water; $535 \mathrm{kcal}$ ) and, eight hours after breakfast, a dinner (steak, salad, dessert; $21 \%$ protein, $49 \%$ carbohydrate, $30 \%$ fat; $561 \mathrm{kcal}$ ) was consumed. During the study volunteers were permitted normal physical activity.

\section{Gammacamera imaging}

Gammacamera imaging started immediately after completion of ingestion of the radiolabelled breakfast with a large field of view gammacamera with a medium energy, parallel hole collimator (GE Starcam, General Electric, Milwaukee, WI). Anterior and posterior images were acquired with the subject erect. For the ${ }^{99 \mathrm{~m} T c}$ counts a $140 \mathrm{keV}$, and for the ${ }^{111} \mathrm{In}$ counts a $245 \mathrm{keV}$, energy window (each with $\pm 20 \%$ window) was utilised. The estimated whole body dose equivalent was $130 \mathrm{~m}$ Rem.

Images were obtained at the following times (hours) after ingestion of the labelled meal, 0 , $0.5,1,2,3-5,8-10,12,24$, and 48 . For each image, two minutes of acquisition were selected. Using variable regions of interest, the radioactivity was quantified in the stomach and ascending colon for ${ }^{99 \mathrm{~m}} \mathrm{Tc}$ and in four regions of the colon (ascending, transverse, descending, rectosigmoid) for ${ }^{111} \mathrm{In}$. The geometric means of the counts obtained from anterior and posterior images were calculated for each region and then corrected for radionuclide decay. The downscatter of ${ }^{111} \mathrm{In}$ into the ${ }^{99 \mathrm{~m} T \mathrm{~T}}$ window was adjusted. For two days, stools were collected and the radioactivity for ${ }^{111}$ In counts was assessed and corrected for decay.

Colonic transit time measured by radio-opaque marker method

Localisation of the radio-opaque markers on the abdominal film taken 24 hours after 
TABLE I Gastric emptying and small bowel transit. Reproducibility studies in healthy women $(n=12)$ and men $(n=20)$

\begin{tabular}{llccc}
\hline Measurement & Sex & Mean & SEM & Median \\
\hline Gastric lag phase (minutes) & F1 & 56 & 7 & 59 \\
& F2 & 56 & 6 & 49 \\
& M1 & 55 & 5 & 57 \\
Gastric half emptying (T1/2 & M2 & 58 & 3 & 58 \\
minutes) & F1 & 202 & 26 & 184 \\
& F2 & 198 & 22 & 201 \\
Small bowel transit (minutes) & M1 & 153 & 9 & 157 \\
& F1 & 163 & 13 & 148 \\
& F2 & 196 & 22 & 179 \\
& M1 & 181 & 23 & 184 \\
& M2 & 196 & 19 & 186 \\
& & & & 190 \\
\hline
\end{tabular}

F1 is the study performed during the follicular and F2 during the luteal phase of the menstrual cycle in women. ingestion of the last radio-opaque marker relied on the bony landmarks and gaseous delineations see on the abdominal $x$ ray. ${ }^{67}$ If no clear contours of the bowel were recognised, markers located to the right of the vertebral spinous processes, above a line from the fifth lumbar vertebrae to the pelvic outlet, were assigned to the right colon. Markers to the left of the vertebral spinous process and above an imaginary line from the fifth lumbar vertebrae to the anterior superior iliac crest were allocated to the left colon. Markers inferior to a line from the pelvic brim on the right and the superior iliac crest on the left were assigned to the rectosigmoid and rectum. ${ }^{67}$ However, if bowel outlines clearly showed a pelvic caecum, an unusual transverse colon or a large sigmoid loop above the fifth lumbar vertebrae, markers were judged to be in the anatomic segment based on the bowel silhouette.

\section{Data analysis}

Gastric emptying by the scintigraphic method was assessed by the gastric lag time, postlag emptying rate, and the half emptying time $\left(\mathrm{T}^{1} / 2\right)$. The gastric lag time ( $\left.\mathrm{min}\right)$ was the time taken for $10 \%$ of the radiolabel to empty from the stomach. ${ }^{12}$ The gastric postlag emptying rate $(\% / \mathrm{min})$ was characterised by the slope
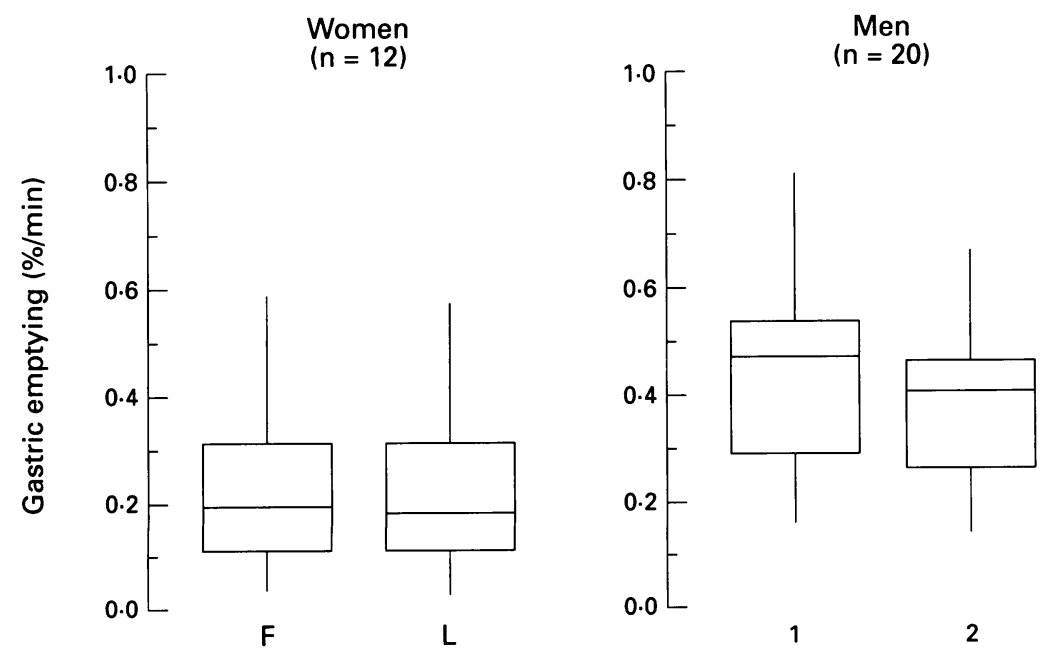

$W v M: p=0.005$

Figure 1: Postlag phase of gastric emptying, shown as median values (bars), interquartile ranges (boxes), and range for men and women. Women were studied in the follicular $(F)$ ranges (boxes), and range for men and women. Women were studied in the follicular Women had slower gastric emptying when evaluated by this index. estimated from linear regression analysis of data from the first point beyond the lag time until the time when $90 \%$ of the radiolabel had emptied from the stomach. ${ }^{42}$

Small bowel transit time was assessed by subtracting the time for $10 \%$ of the radiolabelled breakfast to empty from the stomach from the time taken for $10 \%$ of the label to enter the colon. ${ }^{12} 42$

Colonic transit was evaluated by the geometric centre of counts in the colonic regions of interest (ROI). The geometric centre was the weighted average of the proportions of counts in the four ROI of the colon. ${ }^{13}$ The regions, designated by numbers $1-4$ as weighting factors were, respectively, the ascending, transverse, descending, and rectosigmoid colons. The stool was designated as region 5 . The proportion in each region was multiplied by the weighting factor and the sum calculated. A low geometric centre indicated that most radiolabel was closer to the caecum, whereas a high value indicated that the major part of the radiolabel was closer to the stool.

\section{Colonic transit time measured by radio-opaque marker method}

The total number of all markers for each colonic segment was multiplied by a factor of 1.0 and designated as the mean colonic transit time for that segment. The mean total colonic transit time was the sum of the calculated mean segmental transit times. ${ }^{7}$

Physical activity score, SCL-90-R score, smoking habits, alcohol, and coffee consumption

Assessments of total energy expenditure, expressed as kilocalories/week, ${ }^{40}$ and the psychological symptom scores for primary symptoms and global indices of distress, ${ }^{41}$ were rated. Smoking habits were quantified by pack years of actual consumption, alcohol consumption by units a week ( 1 unit beer, wine or hard drink $=10 \mathrm{~g}$ alcohol), and coffee by cups a day.

\section{Hormone concentrations}

Serum concentrations of progesterone and oestradiol were assessed with enhanced luminescence and radioimmunoassays, respectively. A concentration of progesterone above $2 \mathrm{ng} / \mathrm{ml}$ was regarded as consistent with the luteal phase, values below $0.7 \mathrm{ng} / \mathrm{ml}$ with the follicular phase.

\section{Statistical analysis}

Gastrointestinal transit data were expressed by box whisker plots showing the median, the interquartile, and the total ranges. Normally distributed values were evaluated statistically by paired or unpaired $t$ tests and non-parametric data by the Wilcoxon's signed rank test. When we compared the radio-opaque and scintigraphic methods, we assessed the correlation between both the methods for each study period. Correlations between the results were 


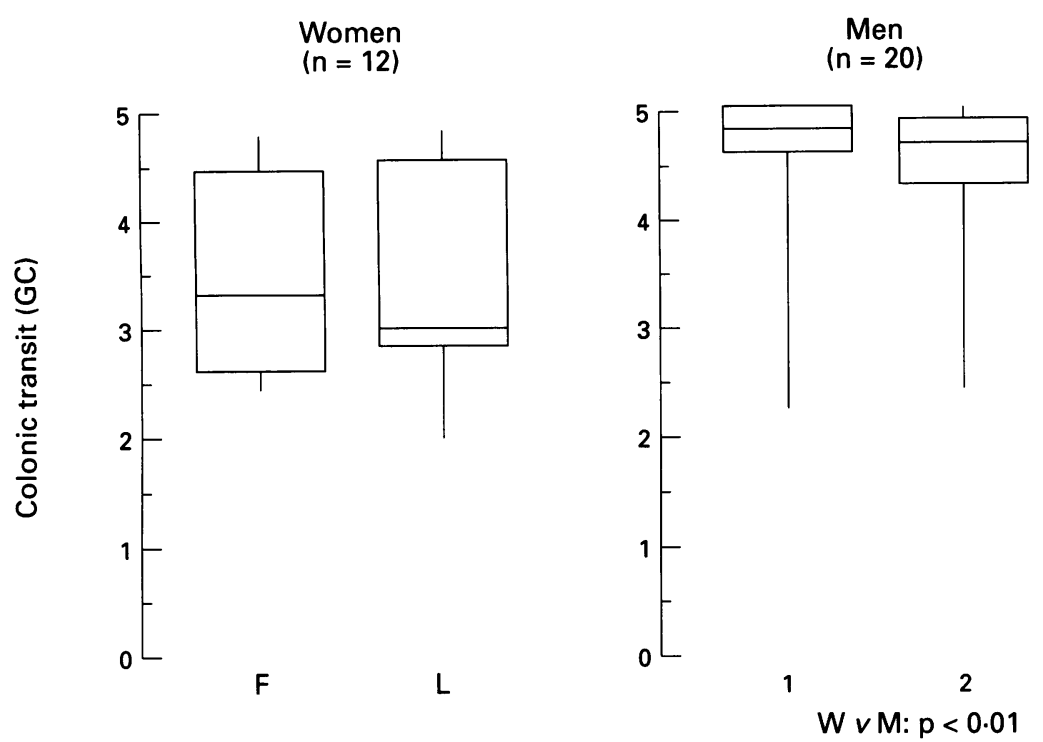

Figure 2: Colonic transit in healthy men and women, expressed as the geometric centre of counts at 48 hours, shown as median values (bars), interquartile ranges (boxes), and range for men and women. Women were studied in the follicular $(F)$ and luteal $(L)$ phases of the menstrual cycle. Men were studied twice, 14 days apart. Colonic transit was significantly faster in men.
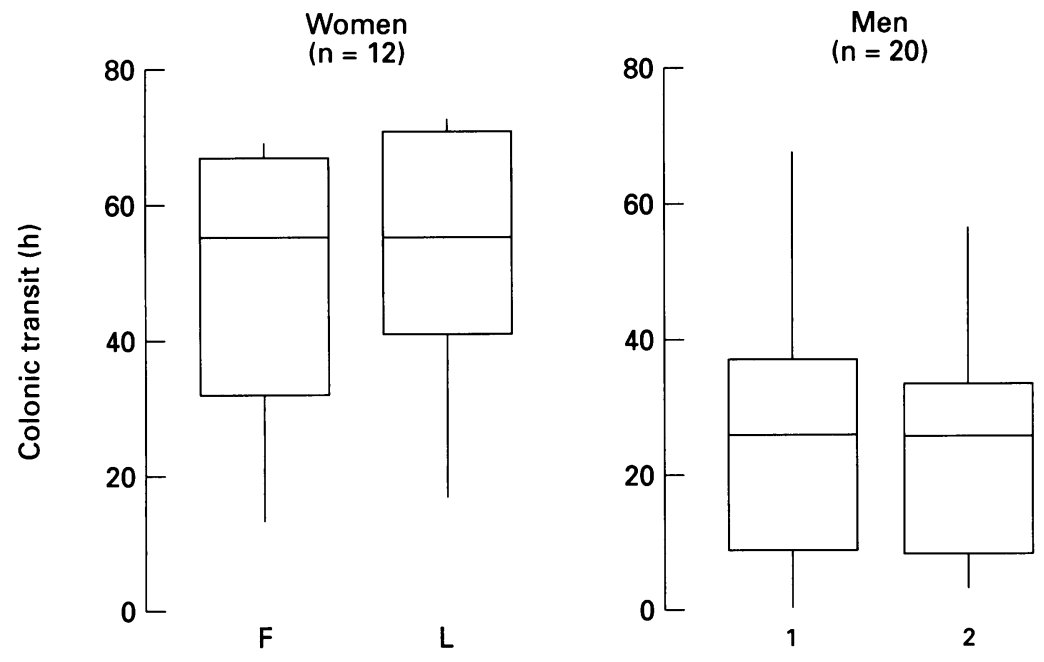

Figure 3: Total colonic transit as measured by the radio-opaque marker method, in healthy men and women, shown as median values (bars), interquartile ranges (boxes), and range for men and women. Women were studied in the follicular $(F)$ and luteal $(L)$ phases of the menstrual cycle. Men were studied twice, 14 days apart. Colonic transit was significantly faster in men at 24 hours.

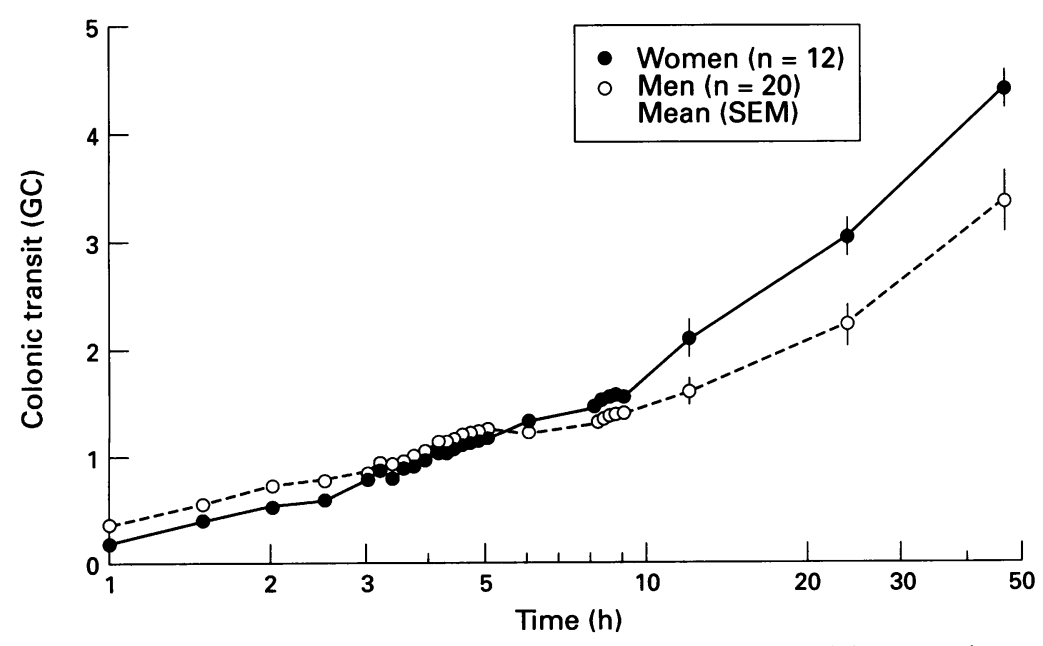

Figure 4: Sex differences in colonic transit, expressed as the progression of the geometric centres of scintigraphic markers against time. High values for the GC, signifying faster colonic transit, became apparent in men after 12 hours (approximately). At that time the $G C$ was approximately 2.0 indicating that the centre of counts was in the transverse colon. based on linear regression analysis. The influence of covariates on gut transit was evaluated using multiple analyses of variance. Significance was declared at $a<0 \cdot 05$.

In women, the indices were compared intra as well as interindividually for the follicular and luteal phase; in men, the first and second studies were compared. As the menstrual cycle had no measurable effect on gastrointestinal transit, for sex comparisons we used the two values for women and the two for men to obtain a single value for each individual.

Intraindividual variability between results at each level of the gut were not significantly different between sexes and consequently have been summarised together.

\section{Results}

CHARACTERISTICS OF THE GROUPS

The mean age in both groups was 29 years (range, women: 19-44; men: 21-45). Although the body mass index did not differ significantly between the sexes (mean (SEM) women: 23.97 (0.95); men: 25.48 (0.63), weight and height were significantly different $(\mathrm{p}<0.001)$. Men were heavier (84 (2) $v 68$ (3) $\mathrm{kg}$ ) and taller (182 (2) $v 168(2) \mathrm{cm})$. Although men ingested slightly more calories, the values were not significantly different between sexes, as well as between the two studies (women: study 1: 2845 (420), study 2: 2562 (288) kcal/day, men: 3384 (570) and 3296 (479) kcal/day). Smoking, coffee, and alcohol consumption were not significantly different between paired studies.

Women had their menarche at a mean age of $13(0.4)$ years, the mean cycle length was 28 $(0.7)$ with a menstrual length of five $(0.3)$ days. The number of pregnancies was one $(0 \cdot 5)$. The fluctuation of hormonal concentrations between the follicular and luteal phases was significant for progesterone and oestradiol $(\mathrm{p}<0.001$ and $\mathrm{p}=0.018$, respectively). Progesterone measured in the follicular phase was $0.57(0.07)$, in the luteal phase $6.07(1.11)$ $\mathrm{ng} / \mathrm{ml}$. Oestradiol concentrations were 79.67 $(11.09)$ and $137.33(16.43) \mathrm{pg} / \mathrm{ml}$, respectively.

\section{SEX DIFFERENCES}

\section{Gastric emptying}

The lag phase for solid meals and the half times for gastric emptying were not significantly different between sexes (Table I). However, the postlag gastric emptying rate (Fig 1) was significantly faster in men than women $(p=0.005)$. No significant change in any of the three indices of gastric emptying was noted during the menstrual cycle (Table I, Fig 1).

\section{Small bowel transit}

The index demonstrated no sex differences and no effect of the menstrual cycle (Table I). The variability in women was similar to that in men. 


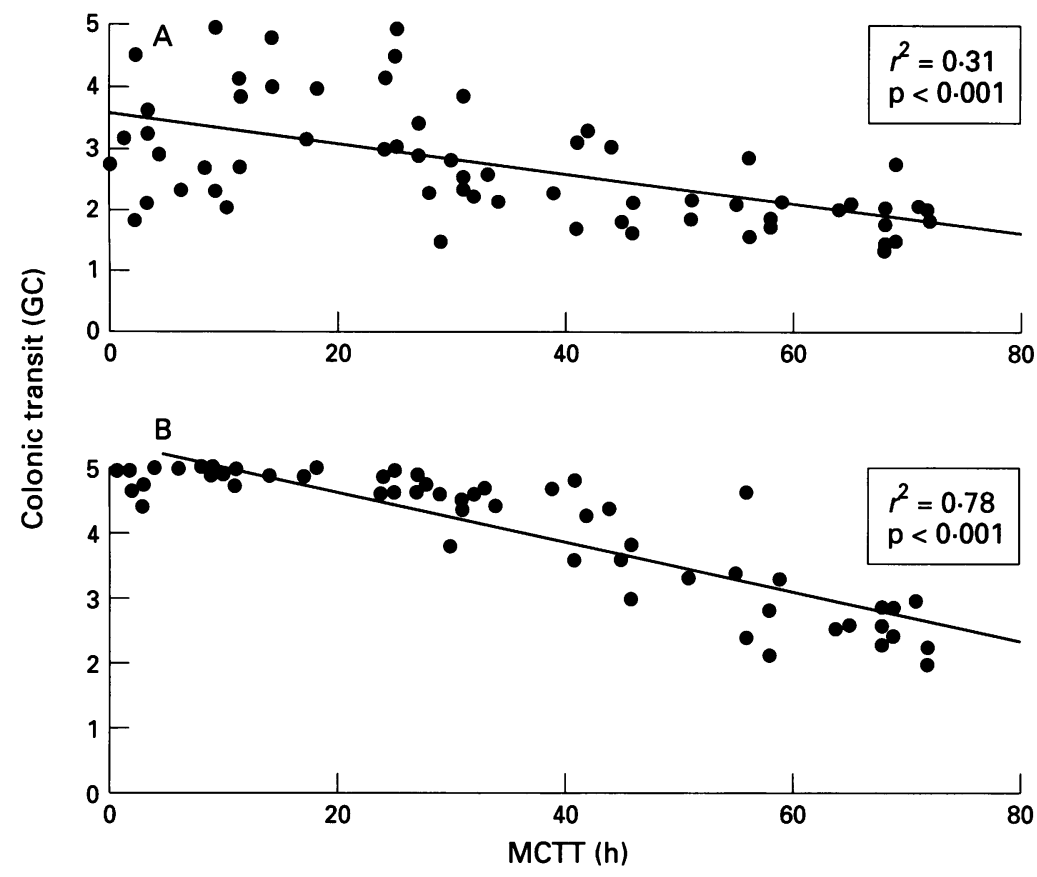

Figure 5: Relations between mean colonic transit time (MCTT) by the radio-opaque marker method and the geometric centres (radioscintigraphic method) at $24(A)$ and 48 (B) hours; 64 observations on 32 people.

\section{Colonic transit}

When expressed as the geometric centre (GC) at six and 24 hours (data not shown) and 48 hours (Fig 2), colonic transit was stable throughout the menstrual cycle. Total colonic transit time (Fig 3), measured by the radioopaque marker method, was also not influenced by the menstrual cycle. However, irrespective of the cycle phase in women, colonic transit was significantly faster in men (Figs 2 and 3).

Mean values of GCs for all available time points were plotted (Fig 4). Sex differences were most pronounced in the later observations, when markers should have reached the distal colon - that is, at 12 hours and beyond. This difference reached statistical significance when the areas under the curves were compared $(\mathrm{p}<0.001)$.

Within individual studies, correlations between the scintigraphic GC at 24 or 48 hours and the results with radio-opaque markers were highly significant $(p<0.001$, Fig 5$)$. Using the average value of the two studies for each individual (data not shown), these correlations were also significant $(p<0 \cdot 005)$.

\section{INTRAINDIVIDUAL VARIABILITY}

Individual differences between study 1 and study 2 for each person were evaluated for each

TABLE II Gastric emptying and small bowel transit. Interindividual variability

\begin{tabular}{llllc}
\hline & \multicolumn{4}{c}{ Difference between replicate studies } \\
\cline { 2 - 5 } Measurement & Sex & Mean delta & Median delta & Range delta \\
\hline Gastric lag phase (minutes) & F & 0 & 2 & 60 \\
Gastric half emptying (T $1 / 2$ & M & 3 & 3 & 36 \\
minutes) & F & 3 & 2 & 66 \\
Postlag slope (0/0 per minute) & M & 10 & 4 & 183 \\
Small bowel transit (minutes) & M & 0.001 & 0.004 & 0.44 \\
& F & 4 & 0.016 & 0.33 \\
& M & 19 & 4 & 211 \\
\hline
\end{tabular}

^Delta refers to the difference between studies 1 and 2 for each person. index of transit. The means and medians of these intraindividual differences were not significantly different from 0 for the gastric lag phase, postlag emptying slope, and $\mathrm{T}_{1}^{1} / 2$ of gastric emptying. However, the ranges of these intraindividual differences were broad. Similar conclusions pertain to small bowel transit (Table II).

Repeated measurements of colonic transit also showed mean results that were very reproducible. When differences between study 1 and study 2 for both sexes were combined, median differences of the GCs at six and 24 hours were very close to zero and the interquartile ranges were also quite narrow (Fig 6). However, outliers were noted and the total ranges of interindividual differences were wide (Fig 7). Radio-opaque marker transit yielded similar comparisons between replicate studies. Figure 7 shows the mean colonic transit times for both studies in all subjects.

\section{Discussion}

Epidemiological findings ${ }^{4344}$ suggest that women are more constipated than are men and Heaton et $a l^{44}$ suggested a hormonal cause, especially in women of childbearing age. It is noteworthy, therefore, that female hormones influence gastrointestinal muscle function in animals. Contractile activity was inhibited in vitro by progesterone ${ }^{45-47}$ and excited by oestradiol $^{14} 48$; moreover, gastrointestinal transit in rodents was influenced by both classes of hormones. ${ }^{15-18}$ To extend these findings and quantify the effects in humans, transit times through the gut were estimated with radioopaque markers 4950 or lactulose $\mathrm{H}_{2}$ breath excretion. ${ }^{51}$ The results are inconsistent. Some have indicated no significant difference between women and men, whether the measurements were those of orocaecal transit ${ }^{52}$ or mouth to anus transit. ${ }^{53}$ Others have suggested a significant prolongation of mean colonic transit in women ${ }^{74-57}$ but were unable to demonstrate changes of transit during the menstrual cycle. ${ }^{56}$

We studied healthy women and men twice under the same standardised conditions, in particular we controlled for diet, alcohol, and coffee consumption, physical activities, and psychological patterns. None of these factors exhibited consistent effects. Physical activity and the psychological patterns did not vary significantly during the menstrual cycle. By preselecting healthy, non-obese volunteers, weight and height, although significantly different between the sexes did not influence transit significantly. We confirmed that hormone concentrations fluctuated predictably during the menstrual cycle, but transit was not systematically different during the phases of the menstrual cycle, even if we excluded from the analysis three women who showed the least changes in circulating levels of progesterone during the luteal phase.

In both sexes, group median (or mean) values were essentially identical for replicate studies. Closer inspection of the results showed that, though group differences 

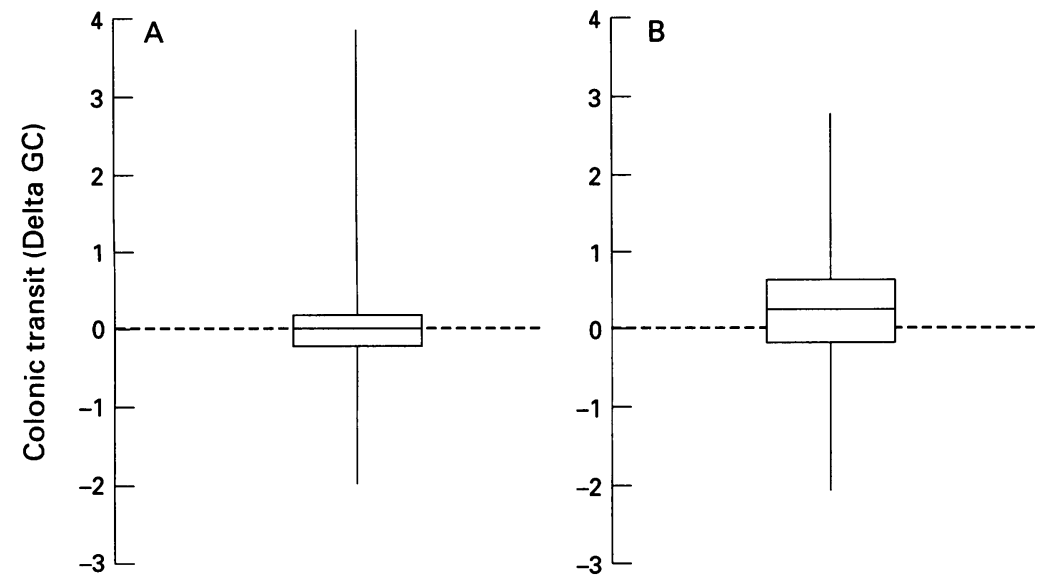

Figure 6: Intraindividual differences for colonic transit, expressed as geometric centres at six $(A)$ and 24 (B) hours, for 12 women and 20 men, each of whom was studied on two occasions. The median value for delta (differences between studies 1 and 2) was close to zero, and half the subjects (interquartile range in the box) yielded very similar values for replicate studies. The entire range was wide, as influenced by several outliers.

between replicate tests (for example, median and interquartile ranges for delta values) were generally small, outliers were recorded. Moreover, the intrinsic variability of transit in men implied that any additional perturbation in women (for example, due to the menstrual cycle) would need to be quite large if it were to be statistically and clinically important. Our sample size provided an $80 \%$ statistical power, at a significance level of 0.05 , to detect a difference of one standard deviation within a person. Based on this index, the menstrual cycle did not change gastrointestinal transit. Thus, though a minor effect of the menstrual cycle on transit cannot be excluded, any changes that might be found with larger samples would be small compared with intrinsic biological variations.

In both follicular and luteal phases, one of three indices of gastric emptying (postlag emptying slope) was significantly slower in women. However, two other indices of gastric emptying ( $\mathrm{T}_{1 / 2}$ and lag phase), and small bowel transit, showed no sex differences. Thus, we feel any effects of sex on gastric emptying and small bowel transit are probably not of great biological significance. The differences between sexes for colonic transit were more impressive and

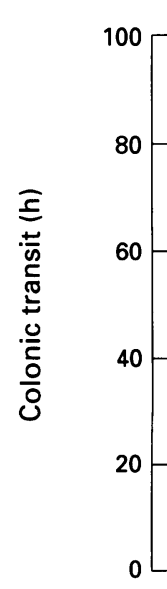

Women

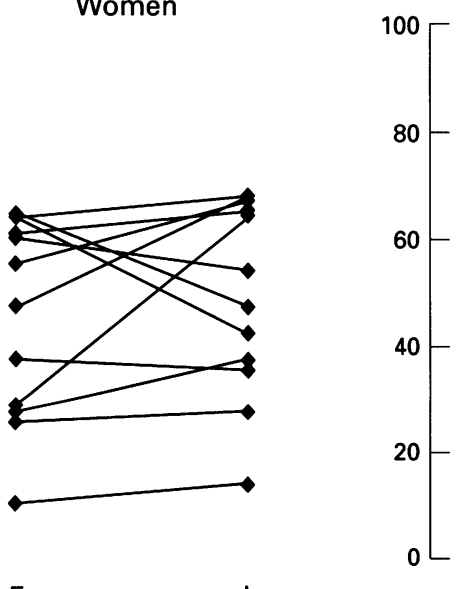

Men

L

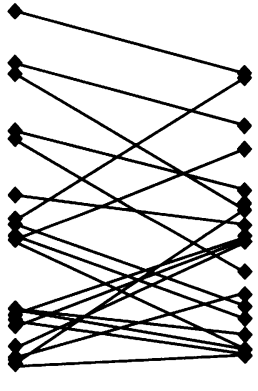

1 consistent with previous studies. ${ }^{7} 202755-57$ The scintigraphic method allowed us to compare GCs at various times, Figure 4 suggests that the sex difference was most obvious in the later scans, when the marker had reached the distal colon.

The reproducibility of group results was impressive. However, in a few persons, intraindividual variabilities were considerable, and these were seen at all levels of the gut. We feel these represent the biological variations within a person, which must be kept in mind when assessing the effects of any intervention, such as treatment with a drug. The magnitude of the intraindividual variabilities in colonic transit were similar for the scintigraphic and radioopaque techniques, suggesting that they reflect physiological changes in gut function, rather than being due to methodological artefacts. Factors that might contribute to this physiological variability include diet, physical activity, sex hormones, weight, height, alcohol, and coffee consumption, as well as psychological factors. We attempted to control for all of these, and none was significant.

In summary, we were unable to demonstrate in healthy women an effect of the menstrual cycle on gastric emptying, small bowel, and colonic transit. Thus, physiological effects of hormones can be disregarded during assessments of transit. Although gastrointestinal symptoms fluctuate during the menstrual cycle, our results imply that these variations should not be caused by changes in transit. One of these indices of gastric emptying showed a sex difference and slower colonic transit in women was again confirmed.

The study was supported in part by a grant from the Swiss National Science Foundation (LPD) and by grants DK 32121 and RR00585 from the National Institutes of Health, Bethesda, MD, USA (SFP)

1 Cann PA, Read NW, Brown C, Hobson N, Holdsworth $\mathrm{CD}$. Irritable bowel syndrome; relationship of disorders in the transit of a single, solid meal to symptom patterns. Gut the transit of a single,

2 Stivland T, Camilleri $M$, Vassallo $M$, Proano $M$, Rath D, Brown $M$, et al. Scintigraphic measurement of regional gut transit in idiopathic constipation. Gastroenterology 1991; 101: 107-15.

3 Vassallo $M$, Camilleri $M$, Phillips SF, Brown $M L$, Chapman NJ, Thomforde GM. Transit through the proximal colon influences stool weight in the irritable bowel syndrome. Gastroenterology 1992; 102: 102-8.

4 LaBrooy SJ, Male PJ, Beavis AK, Misiewicz JJ. Assessment of the reproducibility of the lactulose $\mathrm{H} 2$ breath test as a measure of mouth to caecum transit time. Gut 1983; 24: 893-6.

5 Proano $M$, Camilleri M, Phillips SF, Brown ML, Thomforde GM. Transit of solids through the human colon: regional quantification in the unprepared bowel. Am $\mathcal{F}$ Physiol 1990; 258: G856-62.

6 Arhan P, Devroede G, Jehannin B, Lanza M, Faverdin C, Dornic C, et al. Segmental colonic transit time. Dis Colon Rectum 1981; 24: 625-9.

7 Metcalf AM, Phillips SF, Zinsmeister AR, MacCarty RL Beart RW, Wolff BG. Simplified assessment of segmental colonic transit. Gastroenterology 1987; 92: 40-7

8 Scarpello JHB, Barber DC, Hague RV, Cullen DR, Sladen GE. Gastric emptying of solid meals in diabetics. $B M \mathcal{F}$ 1976; 2: 671-3.

9 Collins PJ, Horowitz M, Cook DJ, Harding PE, Shearman DJC. Gastric emptying in normal subjects: a reproducible technique using a single scintillation camera and comtechnique using a single scintillation

10 Brophy CM, Moore JG, Christian PE, Egger MJ, Taylor AT. Variability of gastric emptying measurements in man employing standardized radiolabeled meals. Dig Dis Sc 1986; 31: 799-806.

11 Camilleri $M$, Zinsmeister AR. Towards a relatively inexpensive, noninvasive, accurate test for colonic motility disorders. Gastroenterology 1992; 103: 36-42.

Figure 7: Mean colonic transit time by the radio-opaque marker method; duplicate studies in 12 women and 20 men. Women were studied in the follicular $(F)$ and luteal $(L)$ phases of the menstrual cycle. Men were studied twice, 14 days apart. 
gastric emptying and small bowel transit. Dig Dis $S c i$ 1991; 36: 609-15.

13 Charles F, Camilleri M, Phillips SF, Thomforde GM Forstrom LA. Scintigraphy of the whole gut: clinical evaluation of transit disorders. Mayo Clin Proc 1995; 70 113-8.

14 Gill RC, Bowes KL, Kingma YJ. Effect of progesterone on canine colonic smooth muscle. Gastroenterology 1985; 88: 1941-7.

15 Datta S, Hey VM, Pleuvry BJ. Effects of pregnancy and associated hormones in mouse intestine, in vivo and in vitro. Pflugers Arch 1974; 346: 87-95.

16 Scott LD, DeFlora E. Intestinal transit in female rats: Effect of pregnancy, estrous cycle, and castration. Gastroenterology 1983; 84: 1303.

17 Ganiban G, Besselman D, Harcelrode J, Murthy SNS Effect of sex steroids on total gastrointestinal transit in male rats. Gastroenterology 1985; 88: 1713 .

18 Ryan JP, Bhojwani A. Colonic transit in rats: effect of ovariectomy, sex steroid hormones, and pregnancy. $A m \mathcal{F}$ Physiol 1986; 251: G46-50.

19 Davies GJ, Crowder M, Reid B, Dickerson JWT. Bowel function measurements of individuals with different eating patterns. Gut 1986; 27 : 164-9.

20 Wald A, van Thiel DH, Hoechstetter L, Gavaler JS, Egler $\mathrm{KM}$, Verm $\mathrm{R}$, et al. Gastrointestinal transit: the effect of the menstrual cycle. Gastroenterology 1981; 80 497-500.

21 Datz FL, Christian PE, Moore J. Gender-related differences in gastric emptying. F Nucl Med 1987; 28: 1204-7.

22 Lawaetz O, Dige-Petersen H. Gastric emptying of liquid meals: a study in 88 normal persons. Ann Chir Gynaecol 1989; 78: $267-76$

23 Hutson WR, Roehrkasse RL, Wald A. Influence of gender and menopause on gastric emptying and motility. Gastroenterology 1989; 96: 11-7.

24 Notivol R, Carrio I, Cano L, Estorch M, Vilardell F. Gastric emptying of solid and liquid meals in healthy young subjects. Scand f Gastroenterol 1984; 19: 1107-13.

25 Bisdee JT, Garlick PJ, James WPT. Metabolic change during the menstrual cycle. Br f Nutr 1989; 61: 641-50.

26 Kamm MA, Farthing MJG, Lennard-Jones JE. Bowel function and transit rate during the menstrual cycle. Gut 1989 30: $605-8$.

27 Wyman JB, Heaton KW, Manning AP, Wicks ACB Variability of colonic function in healthy subjects. Gut 1978; 19: $146-50$.

28 Turnbull GK, Thompson DG, Day S, Martin J, Walker E, Lennard-Jones JE. Relationship between symptoms, menLennard-Jones JE. Relationship between symptoms, menstrual cycle and orocaecal transit in

29 Horowitz M, Maddern GJ, Chatterton BE, Collins PJ Petrucco OM, Seamark R, et al. The normal menstrual cycle has no effect of gastric emptying. $B r f \mathcal{O}$ Obstet Gynaecol 1985; 92: 743-6.

30 Monés J, Carrió I, Calabuig R, Estorch M, Sainz S, Berna $\mathrm{L}$, et al. Influence of the menstrual cycle and of menopause of the gastric emptying rate of solids in female volunteers. Eur f Nucl Med 1993; 20: 600-2.

31 Simmons L, Heitkemper M, Shaver J. Gastrointestina function during the menstrual cycle. Health Care for Women International 1988; 9: 201-9.

32 Whitehead WE, Cheskin LJ, Heller BR, Robinson JC, Crowell MD, Benjamin C, et al. Evidence for exacerbation of irritable bowel syndrome during menses. Gastroenterology 1990; 98: 1485-9.

33 Heitkemper MM, Jarrett M. Pattern of gastrointestinal and somatic symptoms across the menstrual cycle. Gastroenterology 1992; 102: 505-13.

34 Manning AP, Thompson WG, Heaton KW, Morris AF. Towards positive diagnosis of the irritable bowel syndrome. $B M 7$ 1978; 2: 653-4.
35 Basilisco G, Camboni G, Bozzani A, Vita P, Doldi S, Bianchi PA. Orocecal transit delay in obese patients. Dig Dig Sci 1989; 34: 509-12.

36 Horowitz M, Collins PJ, Cook DJ, Harding PE, Shearman DJC. Abnormalities of gastric emptying in obese patients. Int f Obesity 1983; 7: 415-21.

37 Wright RA, Krinsky S, Fleeman C, Trujillo J, Teague E. Gastric emptying and obesity. Gastroenterology 1983; 84: 747-51.

38 Harris JA, Benedict FG. A biometric study of basal metabolism in man. Washington, DC: Carnegie Institution, 1919 (Carnegie Institution of Washington, Publication No 279).

39 Anderson JW, Bridges SR. Dietary fiber content of selected oods. Am f Clin Nutr 1988; 47: 440-7.

40 Lee IM, Paffenbarger RS Jr, Hsieh CC. Time trends in physical activity among college alumni, 1962-1988. Am Epidemiol 1992; 135: 915-25.

41 Derogatis LR, Rickels K, Rock A. The SCL-90 and the MMPI: a step in the validation of new self-report scale. $\mathrm{Br}$ f Psychiat 1976; 128: 280-9.

42 Camilleri $M$, Colemont LJ, Phillips SF, Brown ML Thomforde GM, Chapman N, et al. Human gastric emptying and colonic filling of solids characterized by a new tying and colonic filling of solids characterized
method. Am f Physiol 1989; 257: G284-90.

43 Connell AM, Hilton C, Irvine G, Lennard-Jones JE Misiewicz JJ. Variation of bowel habit in two population samples. BMf 1965; 2: 1095-9.

44 Heaton KW, Radvan J, Cripps H, Mountford RA, Braddon FEM, Hughes AO. Defecation frequency and timing, and stool form in the general population: a prospective study. Gut 1992; 33: 818-24.

45 Bruce LA, Behsudi FM, Danhof IE. Smooth muscle mechanical responses in vitro to bethanechol after progesterone in male rat. $A m$ f Physiol 1978; 235: E422-8.

46 Bruce LA, Behsudi FM. Progesterone effects on three regional gastrointestinal tissues. Life Sci 1979; 25: 729-34.

47 Bruce LA, Behsudi FM. Differential inhibition or regional gastrointestinal tissue to progesterone in the rat. Life $\mathrm{Sc}$ i 1980; 27: 427-34.

48 Bruce LA, Behsudi FM. Increased gastrointestinal motility in vitro following chronic estrogen treatment in male rats. Proc Soc Exp Biol Med 1981; 166: 355-89.

49 Cummings $\mathrm{JH}$, Wiggins HS. Transit through the gut measured by analysis of a single stool. Gut 1976; 17: 219-23.

50 Hinton JM, Lennard-Jones JE, Young AC. A new method for studying gut transit times using radioopaque markers. Gut 1969; 10: 842-7.

51 Levitt MD. Production and excretion of hydrogen gas in man. N Engl f Med 1969; 281: 122-7.

52 Rao SSC, Read NW, Brown C, Bruce C, Holdsworth CD. Studies on the mechanism of bowel disturbance in ulcerative colitis. Gastroenterology 1987; 93: 934-40

53 Evans RC, Kamm MA, Hinton JM, Lennard-Jones JE. The normal range and a simple diagram for recording whole gut transit time. Int $\mathcal{f}$ Colorect Dis 1992; 7: 15-7.

54 Lawson M, Everson GT, Klingensmith W, Kern F Jr. Coordination of gastric and gallbladder emptying after ingestion of a regular meal. Gastroenterology 1983; 85: 866-70.

55 Stephen AM, Wiggins HS, Englyst HN, Cole TJ, Wayman $\mathrm{BJ}$, Cummings JH. The effect of age, sex, and level of intake of dietary fibre from wheat on large bowel function in thirty health subjects. $B r \mathcal{F}$ Nutr $1986 ; 56: 349-61$.

56 Hinds JP, Stoney B, Wald A. Does gender or the menstrual cycle affect colonic transit? Am $\mathcal{F}$ Gastroenterol 1989; 84: $123-6$

57 Meier R, Beglinger C, Dederding JP, Meyer-Wyss B, Fumagalli M, Rowedder A, et al. Influence of age, gender, hormonal status and smoking habits on colonic transit time. Neurogastroenterology and Motility 1995; 7: 235-8. 